\title{
Telemonitoramento da COVID-19 com participação de estudantes de medicina: experiência na coordenação do cuidado em Rio Branco, Acre
}

\author{
Rodrigo Pinheiro Silveira, Osvaldo Leal, Pedro Luan da Silva Soares, Laís Ferreira da Cruz, \\ Isadora Damasceno Mello Modesto, Leandra Novais Batista, Louise Araújo Lambert, Paulo \\ Arthur Rodrigues
}

\section{RESUMO}

No final de janeiro de 2020 a Organização Mundial de Saúde declarou estado de Emergência em Saúde Pública pelo novo Coronavírus (Sars-Cov-2) após reconhecimento da pandemia da doença (COVID-19). O vírus chegou ao Brasil no final de fevereiro e ao Acre no meio do mês de março. Com a paralisação das faculdades, articulou-se uma grande equipe de alunos e professores de medicina em uma parceria com o Núcleo Telessaúde Acre para a realização do Telemonitoramento dos casos de COVID-19 em Rio Branco. O objetivo do artigo é relatar a experiência do Telemonitoramento com ênfase na coordenação do cuidado. A equipe trabalha articulada com a central de Teleconsulta da prefeitura, com as Unidades Básicas e Unidades de Referência e tem atuado no encaminhamento oportuno dos casos para atendimento. Também está articulada com duas equipes de psicologia que dão apoio aos pacientes. Essa experiência tem sido de intenso aprendizado para os estudantes de medicina e de importância central para o enfrentamento à COVID-19 em Rio Branco.

Palavras-chave: Coronavírus; Telemonitoramento; Atenção Primária; Coordenação do cuidado; Estudantes de Medicina.

\section{ABSTRACT}

At the end of January 2020, the World Health Organization declared a state of Emergency in Public Health by the new Coronavirus (COVID-19) after recognition of the disease pandemic. The virus arrived in Brazil in late February and in Acre in the middle of March. With the colleges closure, a large team of medical students and professors was articulated in a partnership with telemedicine program to carry out the Telemonitoring of COVID-19 cases in Rio Branco. The purpose of the article is to report the experience of Telemonitoring with an emphasis on coordination of care. The team works in conjunction with the Teleconsultation center of the city hall, with the Basic Units and Reference Units and has been working in the timely referral of cases for assistance. It is also articulated with two psychology teams that support patients. This experience has been of intense learning for medical students and of central importance for facing COVID-19 in Rio Branco.

Keywords: Coronavirus; Telemonitoring; Primary Care; Care coordination; Medical Students.
Revista da Rede APS 2020

Publicada em: 09/06/2020

DOI:10.14295/aps.v2i2.121

Rodrigo Pinheiro Silveira Osvaldo Leal

Pedro Luan S. Soares

Laís Ferreira da Cruz

(Universidade Federal do Acre, Rio Branco, AC, Brasil)

Isadora D. Mello Modesto Leandra Novais Batista Louise Araújo Lambert Paulo Arthur Rodrigues (Centro Universitário UNINORTE, Rio Branco, AC, Brasil)

Correspondência para: Rodrigo Pinheiro Silveira ropsilveira@gmail.com

Osvaldo Leal ms.osvaldo@uol.com.br

Isadora D. Mello Modesto isadorammodesto@gmail.com 


\section{INTRODUÇÃO}

No dia 31 de dezembro de 2019, a Organização Mundial de Saúde (OMS) foi alertada sobre vários casos de pneumonia em Wuhan, na China. Era uma nova cepa de coronavírus que ainda não havia sido identificada em seres humanos. Em 30 de Janeiro de 2020, a Organização Mundial de Saúde declarou estado de Emergência em Saúde Pública pelo novo Coronavirus (Sars-Cov-2), após reconhecimento da pandemia da doença (COVID-19), causando grande impacto sanitário, econômico e social em praticamente todos os países do mundo (OPAS, 2020).

Os casos de Coronavírus chegaram ao Brasil oficialmente em fevereiro de 2020 e no dia 14 de março foi notificado o primeiro caso no Estado do Acre. Desde então, o número de casos vem crescendo exponencialmente, estando o Estado atualmente em terceiro lugar em incidência, com 688,6 casos por 100 mil habitantes.

Ao longo do mês de março, principalmente a partir do início do isolamento social, houve paralisação das atividades comerciais e educativas, incluindo as aulas dos dois cursos de medicina do Estado, os da Universidade Federal do Acre e do Centro Universitário Uninorte. Aproveitando o momento oportuno, foi articulada uma equipe de professores e alunos em parceria com o Núcleo Telessaúde Acre para a realização de duas ações: o atendimento de teledúvidas (Disque Coronavírus) e o Telemonitoramento.

O telemonitoramento é o acompanhamento sistemático dos casos confirmados, prováveis ou suspeitos em grupo de risco de COVID-19, realizado por uma equipe composta de 180 alunos dos dois cursos, supervisionada por professores médicos das instituições. Trata-se de uma ação realizada em parceria entre as instituições de ensino e as Secretarias Estadual de Saúde e Municipal de Saúde de Rio Branco. Esta ação se iniciou no dia 28 de março, quando o Estado tinha assinalado 23 casos confirmados.
Ao término do mês de maio, dois meses depois, já são 5841 casos no Estado e 3895 na capital, Rio Branco. Hoje essa equipe de Telemonitoramento se concentra no acompanhamento de casos na capital, oferecendo apoio matricial para as equipes do interior.

Os objetivos do Telemonitoramento se concentram nos aspectos clínicos e epidemiológicos dos casos, havendo depois da grande disseminação do vírus em fase de transmissão comunitária, um enfoque maior na evolução clínica das pessoas acometidas pelo vírus. A ação procura então promover a avaliação de risco e gravidade dos pacientes com COVID-19, identificando aqueles que podem permanecer em acompanhamento domiciliar, os que devem ser encaminhadas para teleconsulta ou avaliação presencial em Unidade de Referência, e aqueles que precisam de atendimento de urgência e emergência. Desta maneira atua na coordenação do cuidado dos casos confirmados e suspeitos de COVID-19 ao longo do processo de adoecimento, possibilitando o acesso oportuno aos recursos disponíveis no sistema de saúde.

A coordenação do cuidado é considerada um dos quatro atributos essenciais da Atenção Primária (STARFIELD, 2002), sendo uma ação que possibilita que os pacientes acessem oportunamente o sistema de saúde em seus diversos pontos, de acordo com a necessidade. O Telemonitoramento pode ser um dispositivo importante para acionar os pontos de rede conforme a necessidade, evitando exposição da população e identificando o momento certo para intervenções indicadas conforme a fase de doença da COVID-19.

Entendendo que experiências como essas devem ser difundidas, possibilitando que outros locais possam ter iniciativas semelhantes e que possa haver um diálogo sobre as potencialidades e limites da ação, o objetivo do presente artigo é relatar a experiência do Telemonitoramento da equipe de alunos e professores que atuam junto ao Núcleo 
Lambert, L. A.; Rodrigues, P. A.

Telessaúde Acre, com foco na coordenação do cuidado dos casos de COVID-19.

\section{RELATO DE EXPERIÊNCIA}

Ao longo da instalação da epidemia, o sistema de saúde foi se estruturando. Inicialmente a Secretaria de Estado definiu uma unidade de referência somente para a COVID-19, centralizando o atendimento, ao mesmo tempo em que organizava outros pontos de rede. Com o aumento do número de casos na capital, houve estruturação do fluxo de atendimento do município de Rio Branco (Figura 1), tendo as ações de Telemonitoramento e Teleconsulta papel central nesse fluxo, que de fato coloca toda a rede de Atenção Primária envolvida no enfrentamento à COVID-19.

Figura 01 - Fluxograma de organização do sistema de saúde no enfrentamento à Covid-19.

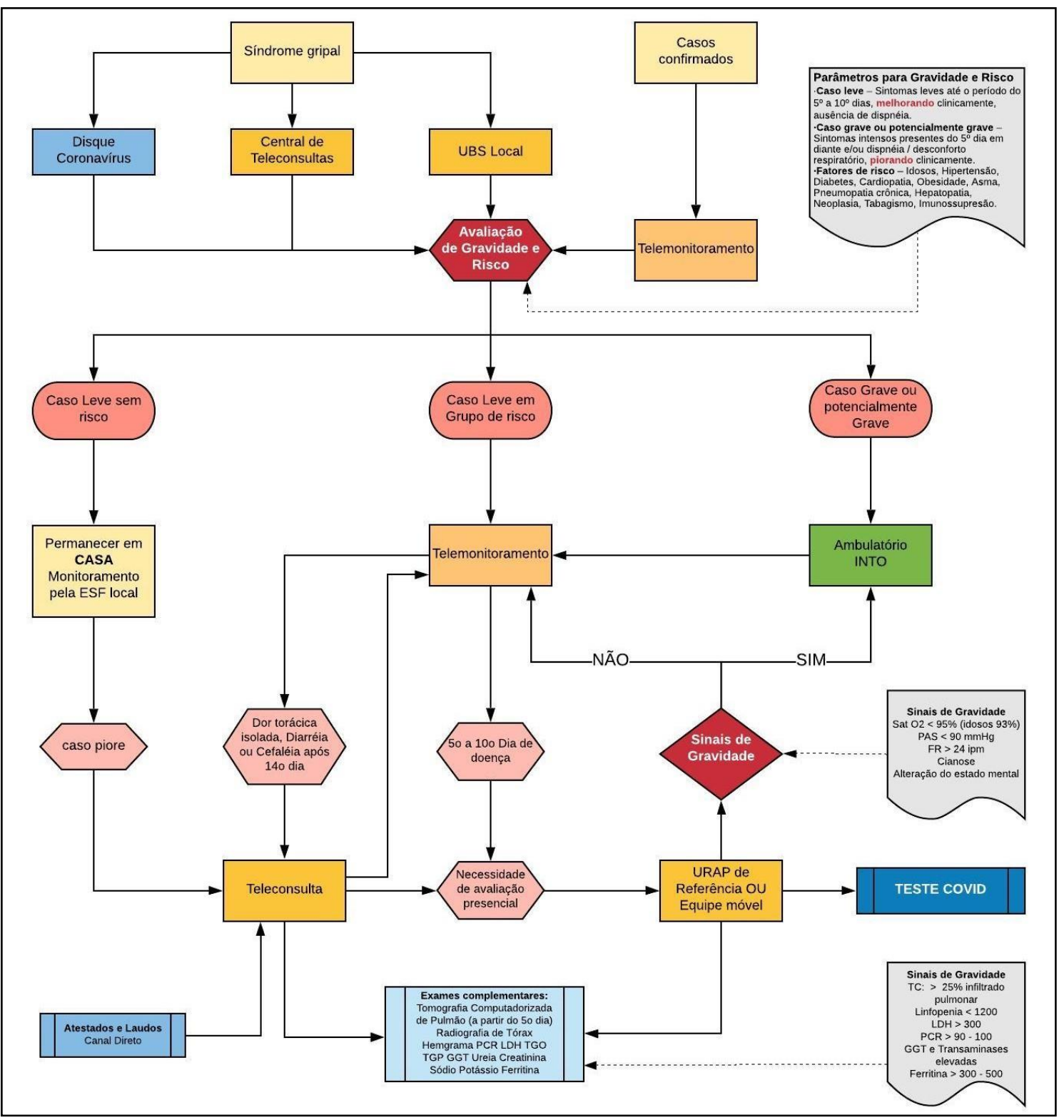

Fonte: Elaborado pela equipe de Telemonitoramento Núcleo Telessaúde Acre 
Entrando na descrição da experiência em si, os casos são captados para Telemonitoramento de 4 maneiras: (1) através das planilhas de casos confirmados repassadas pela Secretaria Municipal de Saúde de Rio Branco (Semsa); (2) identificados a partir do Telemonitoramento (contactantes domiciliares sintomáticos - Casos Prováveis); (3) captados pelo Teledúvidas Disque Coronavírus (Casos Prováveis ou Suspeitos em Grupos de Risco); (4) repassados de outros serviços como a Central de Teleconsultas, as Unidades Básicas e as Unidades de Referência. Os casos são então repassados aos alunos individualmente para se iniciar os contatos.

Para o Telemonitoramento o aluno de medicina utiliza o seu próprio número de telefone/whatsapp, podendo entrar em contato por mensagem de texto ou ligação, a depender das facilidades e dificuldades existentes no caso. Para o início do contato o aluno envia ou lê uma mensagem de apresentação que explica o objetivo da ação, as instituições responsáveis e solicita autorização para o monitoramento. A partir daí os alunos mantém contato periódico com os casos, a depender da gravidade e risco dos pacientes, desta maneira:

-Pacientes em grupo de risco ou
considerados casos graves ou
potencialmente graves - Monitoramento
diário (a cada 24 horas) do início ao fim do
acompanhamento.

- Pacientes com sintomas leves e sem risco - Monitoramento a cada 48 horas até o paciente ficar assintomático. Daí em diante, entrar em contato apenas no 14 은 dia e orientar que se houver algum sintoma eles podem entrar em contato.

Considerando essa premissa, a primeira ação do Telemonitoramento é a avaliação de risco e gravidade, que é realizada conforme a intensidade dos sintomas, evolução do quadro e presença de fatores de risco, conforme exposto abaixo. Caso leve - Sintomas leves a moderados até o período do 5ㅇ a 10을 dias, mas melhorando clinicamente, ausência de dispnéia.

- Caso grave - Sintomas intensos presentes no 5o dia de evolução e/ou dispnéia, desconforto respiratório ou dor torácica, piorando clinicamente.

-Fatores de risco - Idosos, Hipertensão, Diabetes, Obesidade, Cardiopatia, Asma, Pneumopatia crônica, Hepatopatia, Neoplasia, Tabagismo, Imunossupresão.

Essas definições foram baseadas após debate e validação do Protocolo de Manejo da COVID-19 (ACRE, 2020 - dados não publicados), produzido por médicos especialistas do Estado. Conforme a classificação, é seguido o fluxo presente na Figura 2 para coordenação do cuidado e acesso oportuno ao sistema. 
Silveira, R. P.; Leal, O.; Soares, P. L. S.; Cruz, L. F.; Modesto, I. D. M.; Batista, L. N.;

Figura 02 - Fluxograma de ações do Telemonitoramento da COVID-19 em Rio Branco

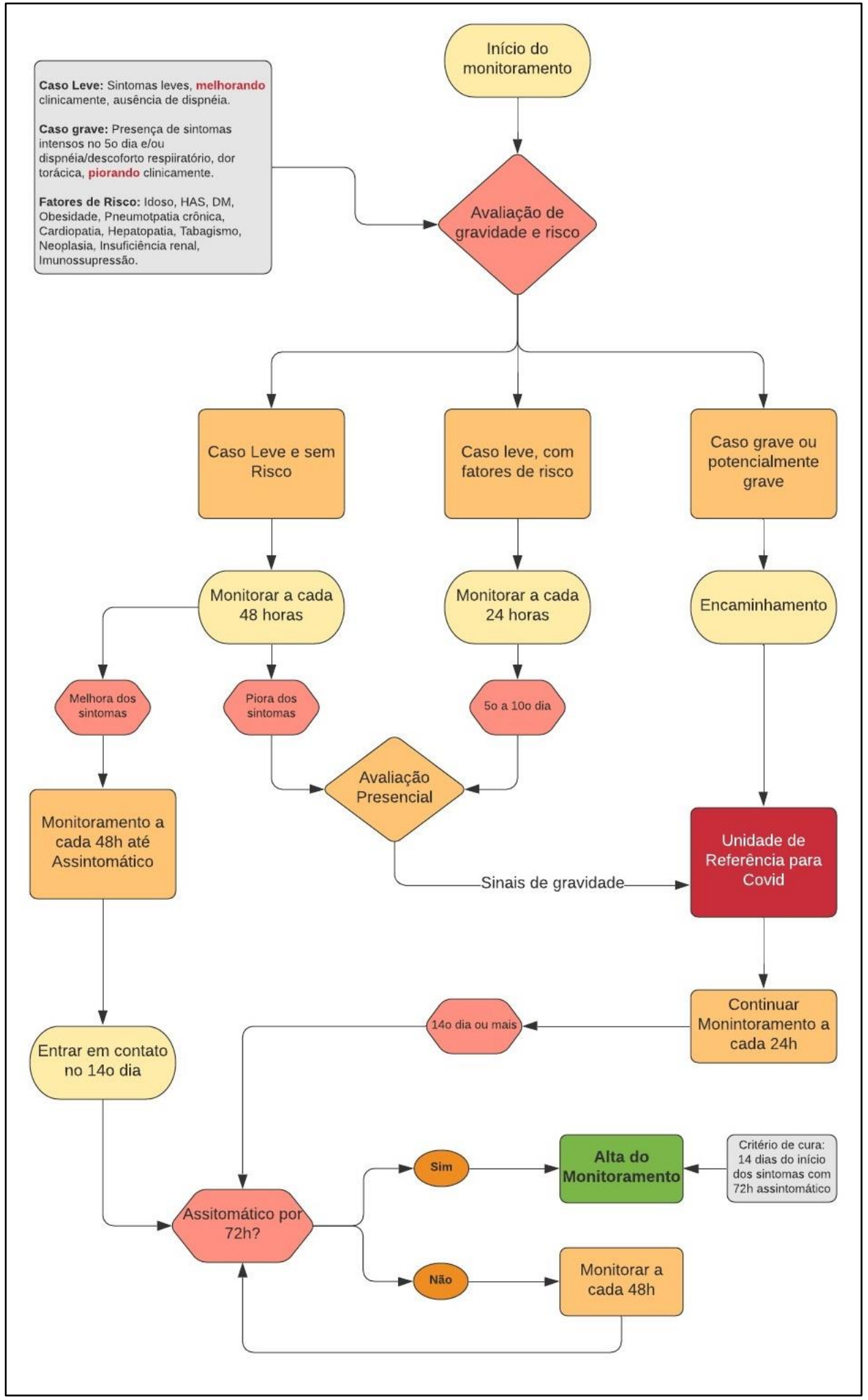

Fonte: Elaborado pela equipe de Telemonitoramento Núcleo Telessaúde Acre 
O registro do monitoramento para acompanhamento dos parâmetros clínicos e epidemiológicas é feito através do preenchimento de um formulário (Google forms) construído especificamente para a ação, seguindo as recomendações do protocolo estadual. É baseado em sessões com encadeamento lógico conforme o fluxo exposto na figura 3. O acompanhamento do monitoramento de casos é todo feito a partir da planilha gerada pelo preenchimento do formulário, possibilitando saber quais pacientes estão evoluindo com gravidade, quais foram encaminhados para a unidade de referência e demais variáveis de acompanhamento

Figura 03 - Encadeamento lógico do formulário de Telemonitoramento da Covid-19 em Rio Branco

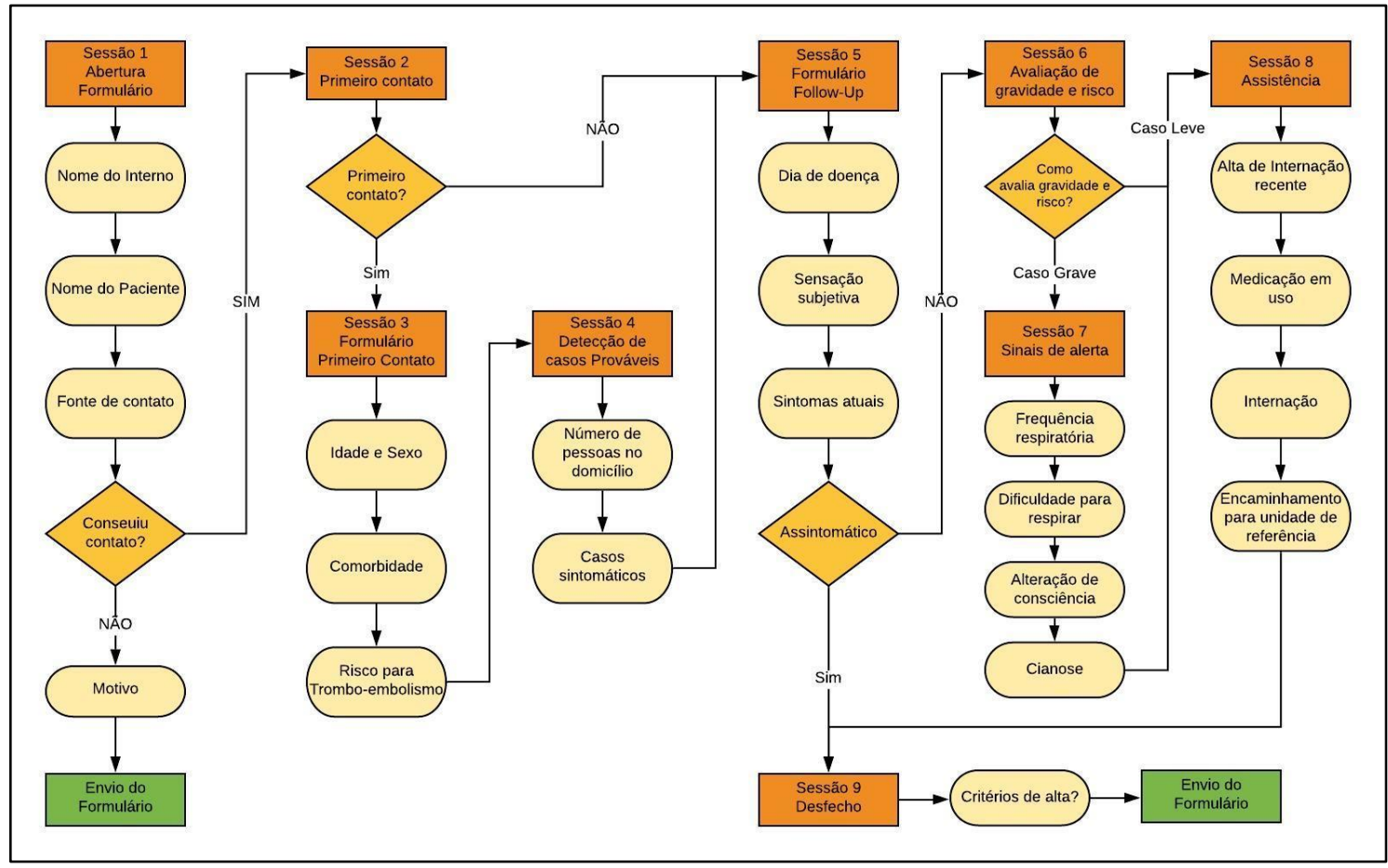

Fonte: Elaborado pela equipe de Telemonitoramento Núcleo Telessaúde Acre

No cotidiano do Telemonitoramento são debatidos os casos com mais risco via whatsapp entre os alunos e os professores orientadores e é promovida então, conforme a necessidade do caso, a coordenação do cuidado.

Em caso de necessidade de encaminhamento para as unidades de retaguarda do telemonitoramento, após contato com o professor orientador, o aluno prepara um resumo do caso contendo as informações a seguir: nome, idade, comorbidade, dia de doença, quadro clínico (sinais e sintomas), medicações em uso, evolução, situação atual e motivo do encaminhamento. Esse resumo é enviado para as referências nas unidades, via Whatsapp ou outro meio necessário, conforme modelo abaixo, que se constitui em um modelo de resumo que deve ser utilizado como parâmetro pelo interno (dados fictícios). 
Figura 04 - Exemplo de resumo do caso para encaminhamento

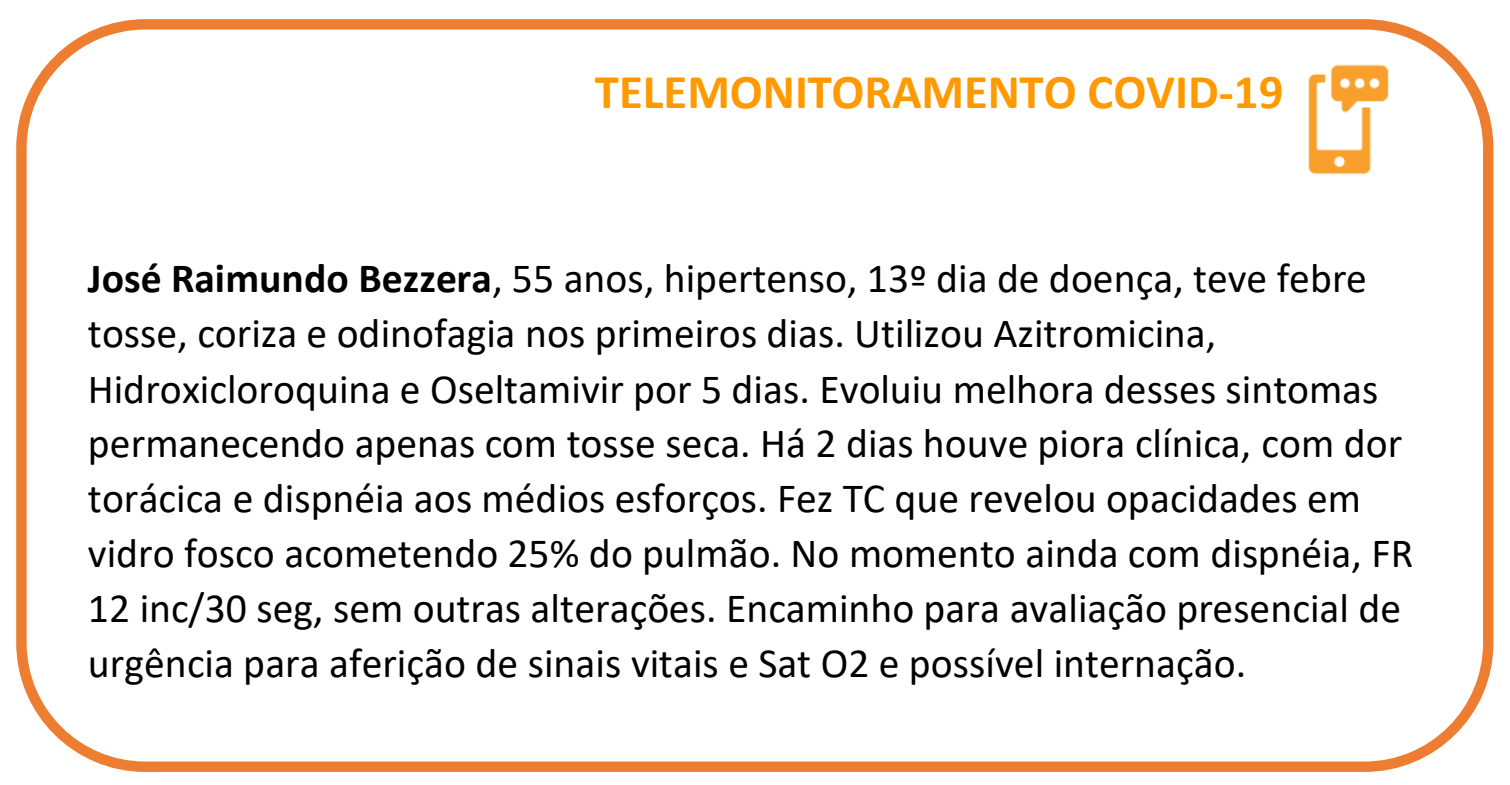

Fonte: Elaborado pela equipe de Telemonitoramento Núcleo Telessaúde Acre

Para pessoas que necessitarem de apoio psicológico, a equipe conta com dois grupos de profissionais da psicologia de referência, que entram em contato com o caso e fazem o atendimento à distância. Essa é uma ação que tem contribuído bastante para que as pessoas acometidas pela COVID-19 possam ter apoio e conforto, pois trata-se de uma situação em que o medo e a ansiedade são bastante comuns.

A alta do monitoramento é alcançada quando o caso preenche o critério de cura clínico especificado na Nota Técnica 03/2020 da Secretaria Estadual de Saúde (ACRE, 2020b) como o tempo de doença de 14 dias ou mais estando assintomático há 72 horas.

Caso seja necessário, é emitido de declaração de alta do monitoramento. Para isso o aluno deverá entrar em contato com o estagiário de referência e fornecer o nome do paciente. Eles então enviam o documento em PDF com o nome do paciente.

Em relação aos dados e à magnitude da ação, até o presente momento foram selecionados 4303 casos para Telemonitoramento, sendo 3892 casos confirmados e foram dadas 1446 altas.

Como apoio aos alunos no Telemonitoramento foi criado um projeto de ensino junto à Universidade Federal do Acre, que consiste na discussão de casos acompanhados, utilizando plataforma virtual, três vezes na semana com professores convidados a cada sessão. Esse suporte possibilita maior aprendizado e a resolução de dúvidas individuais e coletivas sobre o manejo da Covid-19, o telemonitoramento e as ações de coordenação do cuidado. 
Tabela 1 - Números do Telemonitoramento em Rio Branco (Acre) de 30 de maio de 2020

\begin{tabular}{lccc}
\hline & $\mathrm{N}$ & $\%$ & Boletim* $^{*}$ \\
\hline Total de casos selecionados & 4303 & 100 & 4020 \\
Casos confirmados & 3892 & 91,3 & 1309 \\
Casos prováveis e suspeitos & 411 & 8,7 & 114 \\
Altas & 1446 & 33,6 & 1,8 \\
Óbitos & 79 & 6,2 & \\
Recusa ou contato errado & 266 & & \\
\hline
\end{tabular}

*Dados do Boletim Epidemiológico do Estado do Acre em 30 de maio de 2020

Fonte: Elaborado pela equipe de Telemonitoramento Núcleo Telessaúde Acre

\section{DISCUSSÃO}

Pensando na APS, ter uma equipe de Telemonitoramento robusta e qualificada como essa pode contribuir bastante no acompanhamento dos casos e no acesso às ações e unidades de referência, conforme risco, gravidade e fase de doença, bem como necessidade de suporte multiprofissional.

A APS, como primeiro estrato dos níveis de atenção, insere-se no SUS como ordenadora do cuidado e é de importância fundamental para a aplicação de ferramentas complexas de cuidado no território afim de lidar não só com o impacto gerado na dinâmica social da comunidade, mas principalmente na articulação das medidas de controle e prevenção de doença epidêmicas. As UBS dispõem de equipes multiprofissional que pode individualizar àquele território atendido a forma como se conduz o enfrentamento à COVID-19.

Cada componente da APS tem papel fundamental na estruturação do cuidado, nas equipes de saúde da família temos os agentes comunitário de saúde, que vinculado àquela comunidade de forma íntima, pode ter protagonismo nas ações domiciliares de cuidado e monitoramento dos doentes, na contenção da transmissão levando em conta a dinâmica social estabelecida naquele lugar.

Além disso, a educação em saúde é também competência da APS e no cenário da pandemia é tão útil quanto o cuidado complexo, pois é na capacidade de informação e promoção do cuidado no território que estão centradas as possibilidades de sucesso no menor índice de transmissão da doença por repassar de forma íntima a importância das medidas preventivas.

Nesse contexto, o cuidado centrado na pessoa e na particularidade do território faz da APS o estrato competente para o atendimento como verdadeira linha de frente, realizando triagem aqueles que precisam de suporte secundário ou não e o amparando na vigência de sintomatologia leve e com necessidade de suporte mínimo.

O manejo clínico da Síndrome Gripal na APS difere-se avaliando a gravidade dos casos. Em casos leves são inclusas medidas de suporte e conforto, isolamento domiciliar e monitoramento até alta. Para casos graves estão inclusas estabilização clínica e o encaminhamento e transporte a centros de referência ou serviço de urgência/emergência ou hospitalares. A APS tem papel de resolução mediante casos leves e com identificação precoce, sendo responsável pelo 
encaminhamento rápido e correto dos casos graves (BRASIL, 2020).

Nessa experiência é possível observar a coordenação do cuidado como atributo essencial no seguimento e direcionamento dos casos. Considerando que "a coordenação envolve a continuidade de informação dentro do sistema, seja pela continuidade do profissional, seja via prontuário médico" (GUSSO et al., 2019) verificamos diferentes aspectos da coordenação do cuidado em torno da metodologia de acompanhamento dos casos e como norteadora do projeto.

Destaca-se o contato frequente (a depender da classificação de gravidade) e oportuno dos acadêmicos com os pacientes durante o período de duração da doença e a utilização de um instrumento elaborado para uniformização de dados e registro de informações, utilizado como formulário de apoio, que possibilita a supervisão dos casos pelos professores. Além disso, importante destacar a possibilidade de referenciar casos, sendo os principais motivos: administrativos (atestados e laudos), complicações a partir da doença, agravamento do quadro clínico, necessidade de (re)avaliação presencial e até mesmo direcionamento preciso para resolução de quadros clínicos preexistentes e suporte psicológico.

Dentre os desafios estão a existência de algumas lacunas na integração dos serviços, a vulnerabilidade social (pacientes que não conseguem ser inseridos a plataforma de avaliação por indisponibilidade de acesso a dispositivos que permitam o contato ou que não tem a possibilidade de isolamento adequado devido às condições de moradia) e a disponibilidade e estratégias de testagem para captação de casos.

O Telemonitoramento tem sido uma ferramenta amplamente utilizada para acompanhamento dos casos positivos de COVID-19. Ele permite aos alunos uma boa avaliação do estado de saúde dos doentes, mesmo à distância, e funciona como forma de triagem, para diminuir a procura desnecessária das unidades de saúde, o que reflete no melhor atendimento àqueles que realmente necessitam. Funciona também como forma de vínculo entre os profissionais de saúde e o doente, que se sente assistido pelo contato e menos inseguro por estar com uma doença ainda pouco conhecida.

Algumas experiências na pandemia COVID-19 já foram relatadas, como é o caso do projeto integrado entre a Unidade Básica de Saúde Santa Cecília, o Hospital de Clínicas de Porto Alegre e a UFRG, que utilizou os acadêmicos dos cursos da área da saúde para transformação do fluxo de atendimento dentro da comunidade em que abrange, fortalecendo a relação com aquela população nesse momento tão difícil. É válido destacar que papel dos alunos no atendimento foi essencial, visto que o serviço não poderia ter sido ampliado e se tornado eficiente sem a presença destes (MENDONÇA ET AL., 2020).

Já no Pará, a Secretaria de Estado de Saúde Pública firmou uma parceria com o Hospital Albert Einstein para telemonitoramento de pacientes, em que todos com teste positivo que não estão hospitalizados recebem um contato de médicos pneumologistas ou enfermeiros do referido hospital para avaliação do quadro, após consulta primária realizada em qualquer unidade de saúde do estado (VILLANOVA, 2020). Analisando por esse ângulo, consegue-se perceber o quão enriquecedor pode se tornar a telemedicina, propiciando atendimento de qualidade mesmo com a distância entre profissional de saúde e paciente.

O papel do interno de medicina, muito além de apenas passivo na aquisição dos conhecimentos da área, toma um protagonismo nas discussões acerca da educação médica nesse período de pandemia da COVID-19 (FERREL; RYAN, 2020). A sua posição de acadêmico, contrasta com a vivência prática como clínico em treinamento, capacitado, sob devida supervisão, a atuar no cuidado dos pacientes e da comunidade em que está inserido no contexto de estágio (SOLED et al., 2020). 
Porém, durante a crise dos serviços de saúde, instaurada pela disseminação do Coronavírus, novas formas de interação desse indivíduo com a realidade de ensino, aprendizagem e prática na qual está inserido começam a ser adotadas, como forma de sobrepujar as limitações enfrentadas. Nesse contexto, a telemedicina e o telemonitoramento são adotados como uma forma do aluno continuar prestando serviço à comunidade, ajudando a população afetada pela COVID-19, sem exposição dirata ao risco de contrair essa nova doença.

O Telemonitoramento tem contribuído significativamente com a organização do enfrentamento à pandemia de Covid-19 no Acre, possibilitando, através de uma parceria bem sucedida entre Núcleo Telessaúde e os cursos de medicina, um intenso aprendizado sobre o contexto atual de pandemia, bem como o desenvolvimento de uma efetiva resposta à sociedade, o que demonstra sua importância para o Estado e o País, além da efetiva responsabilidade social dessas instituições. 


\section{NOTAS E REFERÊNCIAS}

BRASIL. Ministério da Saúde. Protocolo de Manejo Clínico do Coronavírus (COVID-19) na Atenção Primária à Saúde - Versão 9. Brasília:Ministério da Saúde, 2020

GUSSO, Gustavo; LOPES, José MC, DIAS, Lêda C, organizadores. Tratado de Medicina de Família e Comunidade: Princípios, Formação e Prática. Porto Alegre: ARTMED, 2019.

FERREL, M. N.; RYAN, J. J. The Impact of COVID-19 on Medical Education. Cureus, v. 12, n. 3, p. 10-13, 2020.

MENDONÇA, C. S.; ROSSET, I.; GONÇALVES, M.R. et al. Resposta assistencial de um serviço docente assistencial de APS à pandemia da COVID-19. APS EM REVISTA, v. 2, n. 1, p. 33-37, 2020.

ORGANIZAÇÃO PAN-AMERICANA DE SAUDE (OPAS BRASIL). Folha informativa - COVID-19. Disponível em:

<https://www.paho.org/bra/index.php?option=com_content\&view=article\&id=6101: covid19\&/temid=8 75> Acesso em 30 de maio de 2020

SOLED, D; GOEL, S; BARRY, DANIKA; et al. Medical Student Mobilization During A Crisis: Lessons From A COVID-19 Medical Student Response Team. Academic medicine : journal of the Association of American Medical Colleges, April 8, p. 17-20, 2020. Doi: 10.1097/ACM.0000000000003401

STARFIELD, B. Atenção Primária: equilíbrio entre necessidades de saúde, serviços e tecnologia. Brasília: UNESCO:Ministério da Saúde, 726p. 2004

VILLANOVA, R. Pacientes com Covid contam com Telemonitoramento do Hospital Albert Einstein. Disponível em: http://www.saude.pa.gov.br/2020/04/24/pacientes-com-covid-19-contam-comtelemonitoramento-do-hospital-albert-einstein/. Acesso em 30 de maio de 2020 